\title{
OCORRÊNCIA E DIVERSIDADE ESTRUTURAL DE METABÓLITOS FÚNGICOS COM ATIVIDADE ANTIBIÓTICA
}

\author{
Jacqueline Aparecida Takahashi* e Esther Maria Ferreira Lucas \\ Departamento de Química, Instituto de Ciências Exatas, Universidade Federal de Minas Gerais. Avenida Antônio Carlos, 6627, \\ 31270-010 Belo Horizonte - MG, Brasil
}

Recebido em 21/5/07; aceito em 17/12/07; publicado na web em 17/9/08

\begin{abstract}
OCCURRENCE AND STRUCTURAL DIVERSITY OF FUNGAL METABOLITES WITH ANTIBIOTIC ACTIVITY. Several reasons motivated the development of new generations of antibiotics, such as their high ability to develop resistance to virtually all kinds of anti-infective agents and the crescent market demand for new drugs to treat special demanding patients. After penicillin discovery, several antibiotics were developed from fungal metabolites, since antibacterial secondary metabolites consists on a fungal endogenous protective mechanism against natural competitors. The aim of this review is to present the structural diversity of antibacterial and antifungal metabolites produced by fungi, mentioning sources of fungal isolates, cultivation process and details on the scope of their antibiotic activity.
\end{abstract}

Keywords: fungal metabolites; antibiotics; fermentation.

\section{INTRODUÇÃO}

A primeira referência sobre metabólitos fúngicos parece ter sido publicada em 1911, ${ }^{1}$ mas em menos de 100 anos, até o segundo trimestre de 2007, haviam sido cadastradas nos bancos de dados Caplus e Medline 11376 publicações sobre este tema, inclusive patentes, evidenciando o crescente interesse por esta área.

Dentro deste contexto, uma grande vantagem da prospecção química de metabólitos fúngicos em relação às demais fontes é o fato de que microrganismos podem ser cultivados em larga escala em fermentadores, não havendo prejuízo ao ecossistema, como pode ocorrer com a retirada de plantas e algas de áreas naturais, nem problemas éticos como os que podem advir da prospecção de metabólitos bioativos a partir de insetos, anfíbios e outras espécies animais.

\section{FUNGOS E ANTIBIÓTICOS}

Os primeiros relatos do uso de antibióticos pelo homem são muito antigos, como a descrição do uso de sapatos mofados por chineses para curar feridas infeccionadas nos pés (3000 anos a.C.), porém, o primeiro metabólito fúngico de notória eficácia foi, sem dúvida, a penicilina, substância produzida pelo fungo Penicillium chrysogenun, cuja capacidade de inibir o crescimento bacteriano foi descoberta acidentalmente por Fleming, em 1928. Seu emprego em larga escala no início da década de 40, fruto dos esforços dos pesquisadores ingleses Forey e Chain, levou à redução do índice de mortandade de soldados de $39 \%$ durante a Primeira Guerra Mundial para 3,9\%, na Segunda Guerra. O grande impacto do uso penicilina motivou sua produção industrial, sendo este o primeiro medicamento produzido em grande escala, originando, portanto, a indústria farmacêutica. ${ }^{2}$ Deu-se início, com esta descoberta, à exploração dos microrganismos como fonte de substâncias biologicamente ativas, com uma grande ênfase, ainda vigente, na busca de novas substâncias com atividade antibiótica.

O termo antibiótico foi introduzido por Paul Vuillemin, em 1889, mas foi Waksman quem definiu antibióticos como sendo substâncias produzidas por microrganismos com a capacidade de inibir o cresci-

*e-mail: jat@qui.ufmg.br mento de outros microrganismos ou mesmo destruí-los em soluções diluídas. ${ }^{3}$ Atualmente, o conceito de antibiótico foi expandido para se tornar mais inclusivo, pois diversas substâncias de origem sintética, derivados miméticos de produtos naturais, além de metabólitos secundários de plantas têm apresentado atividade antimicrobiana.

Na década de 70, ocorreu uma expansão do uso dos antibióticos devido à descoberta de substâncias com maior espectro de ação. Além disto, com a descoberta de novas classes de antibióticos ( $\beta$-lactamas, cefalosporinas, macrolídeos, tetraciclinas e amino-glicosídeos), conseguiram-se medicamentos mais potentes, com melhor farmacocinética e maior segurança relacionada ao seu uso (menos efeitos colaterais). A descoberta das cefalosporinas, por exemplo, foi extremamente importante já que estas não causavam descolorimento de dentes em crianças, efeito colateral típico das tetraciclinas, nem geravam tanta resistência, como as penicilinas. ${ }^{4}$

A descoberta destes diversos antibióticos pós-penicilina levou a um êxito tão grande no combate às doenças infecciosas que, na década de 80 , se acreditava que a guerra contra as infecções estava ganha, resultando em um declínio da pesquisa nesta área por parte das indústrias farmacêuticas. Na década seguinte, o avanço das técnicas de triagem robotizadas de grande porte (High Throughput Screening), associadas à disponibilidade de volumosos bancos de moléculas resultantes da química combinatória, colaborou ainda mais para o declínio na busca de novos antibióticos, principalmente a partir de fontes naturais. ${ }^{5}$ Entretanto, alguns fatores têm levado a um retorno do interesse em novas classes de substâncias com atividade antibiótica, como, por exemplo, surgimento de novos alvos bacterianos ou evolução de doenças infecciosas nos últimos 20 anos (Escherichia coli O157:H7, Helicobacter pylori, Ebola, Hantavirus); desenvolvimento de resistência aos antibióticos existentes pelas bactérias patogênicas, fato diretamente ligado ao extenso uso destes medicamentos, muitas vezes sem prescrição médica, em subdosagens ou por um período de tempo insuficiente; toxicidade de alguns antibióticos em uso clínico, como é o caso da gentamicina, que apresenta elevada nefrotoxicidade e ototoxicidade, limitando seu uso; custo elevado da antibioticoterapia, principalmente para pessoas de baixa renda, que costumam ser mais susceptíveis a doenças infecciosas por estarem freqüentemente expostas a condições sanitárias precárias; demanda 
de novas classes de pacientes ou de melhor adequação da antibioticoterapia a indivíduos imunossuprimidos, portadores do vírus HIV, idosos, recém-nascidos, alérgicos etc.

Dentre todos os fatores que justificam a necessidade por novas classes de antibióticos, talvez o mais sério seja a demanda por novos agentes antifúngicos, mais eficientes e de ação mais rápida, uma vez que existem estimativas que mostram que cerca de $40 \%$ de todas as mortes por infecções hospitalares nos últimos 20 anos tenham sido causadas por fungos. ${ }^{6}$

Entretanto, o uso de antibióticos pelo homem não se limita ao tratamento de infecções humanas. Substâncias com atividade antibiótica também têm amplo emprego na agricultura, em especial, os antibióticos com atividade fungicida, pois prejuízos econômicos devido a perdas de lavouras, de grãos e frutas armazenados, causados pela presença de fungos, são preocupantes. Por exemplo, o fitopatógeno Penicillium sclerotigenum parece ter uma ampla capacidade de se adaptar a uma gama de plantas hospedeiras, podendo contaminar, por exemplo, pêras e maçãs com patulina (1), um metabólito secundário com atividade mutagênica e carcinogênica em animais. ${ }^{7}$ Problemas como o crestamento gomoso (causado pelo fungo Didymella bryoniae) do caule de melancia (Citrullus lanatus) têm sido endereçados com a triagem de extratos fúngicos, já que já foram detectadas cepas resistentes ao controle químico atualmente em uso. ${ }^{8}$ No Japão, há relatos de triagem de isolados fúngicos para o combate de infecções de pêras pelo fitopatógeno Alternaria alternata. ${ }^{9}$ Estes isolados mostraram-se capazes de inibirem a formação de lesão e de impedir, em menor proporção, a germinação de esporos.

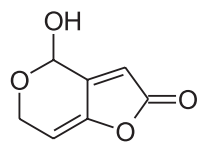

1

Embora haja uma tendência à associação da produção de antibióticos a fungos, diversas bactérias produzem antibióticos importantes, ${ }^{10}$ como mostra a Tabela 1.

Os antibióticos $\beta$-lactâmicos ácido clavulâmico (18), amoxicilina (19), cefaclor (20) e cefriaxona (21), ainda no ano de 1994, atingiram o topo da lista dos medicamentos mais vendidos no mundo. ${ }^{11}$ Embora diversos antibióticos disponíveis hoje no mercado sejam produtos de síntese ou semi-síntese, a maioria das classes de fármacos antibióticos usados na terapêutica atual teve, como modelos, produtos naturais de microrganismos. ${ }^{12}$

\section{DIVERSIDADE ESTRUTURAL DE METABÓLITOS FÚNGICOS COM ATIVIDADE ANTIBIÓTICA}

Os fungos podem ser classificados de acordo com o local de onde são isolados e com a relação que têm com seu hospedeiro. Algumas das classes que mais têm fornecido espécies produtoras de metabólitos de interesse são endófitos, fitopatogênicos, entomopatogênicos, rizosféricos, leveduriformes, de solo e isolados de organismos marinhos, embora fungos mais abundantes na natureza, produtores de esporos e de fácil cultivo, sejam classicamente mais estudados, independente da classe a que pertencem.

Fungos do gênero Penicillium e Aspergillus, conhecidos por sua ubiqüidade e caracterizados geralmente pela formação abundante de esporos, são amplamente estudados. Alguns metabólitos secundários, como o ácido micofenólico (22) ativo contra bactérias Gram-positivas, são produzidos por várias espécies deste gênero (P. brevicompactum, $P$. paxilli, $P$. olivicolor, $P$. canescens, $P$. roqueforti, $P$. viridicatum, $P$. rugulosum e $P$. expansum) sendo, portanto, considerados marcadores em algumas espécies. ${ }^{13}$ Outros gêneros, como o $P$. tulipae, possuem
Tabela 1. Origem das classes de fármacos antibacterianos disponíveis no mercado

\begin{tabular}{|c|c|c|c|}
\hline $\begin{array}{l}\text { Desco- } \\
\text { berta }\end{array}$ & Antibacteriano & Classe & Fonte \\
\hline 1929 & Penicilina G (2) & $\beta$-lactâmico & Penicillium $s p$ \\
\hline 1932 & Sufapiridina (3) & Sulfonamida & síntese \\
\hline 1944 & Estreptomicina (4) & $\begin{array}{l}\text { Aminoglico- } \\
\text { sídeo }\end{array}$ & $\begin{array}{l}\text { Steptomyces } \\
\text { griseus }\end{array}$ \\
\hline 1945 & Cefalosporina (5) & $\beta$-lactâmico & Cephalosporium \\
\hline \multicolumn{4}{|l|}{$s p$} \\
\hline 1947 & Cloranfenicol (6) & $\begin{array}{l}\text { Fenilpropa- } \\
\text { nóide }\end{array}$ & $\begin{array}{l}\text { Streptomyces } \\
\text { venesuelae }\end{array}$ \\
\hline 1948 & Cloritetraciclina (7) & Tetraciclina & $\begin{array}{l}\text { Streptomyces } \\
\text { rimosus }\end{array}$ \\
\hline 1950 & Eritromicina (8) & Macrolídeo & $\begin{array}{l}\text { Streptomyces } \\
\text { erythreus }\end{array}$ \\
\hline 1955 & Vancomicina (9) & $\begin{array}{l}\text { Glicopep- } \\
\text { tídeo }\end{array}$ & $\begin{array}{l}\text { Streptomyces } \\
\text { orientalis }\end{array}$ \\
\hline 1955 & Virginaminicina (10) & $\begin{array}{l}\text { Streptogra- } \\
\text { mina }\end{array}$ & $\begin{array}{l}\text { Streptomyces } \\
\text { virginae }\end{array}$ \\
\hline 1955 & Amfotericina (11) & Polieno & $\begin{array}{l}\text { Streptomyces } \\
\text { nodosus }\end{array}$ \\
\hline 1955 & Lincomicina (12) & Licosamida & $\begin{array}{l}\text { Streptomyces } \\
\text { lincolnensis }\end{array}$ \\
\hline 1959 & Rifamicina (13) & Ansamicina & $\begin{array}{l}\text { Streptomyces } \\
\text { mediterranei }\end{array}$ \\
\hline 1962 & Ácido nalidixico (14) & Quinolona & síntese \\
\hline 1969 & Fosfomicina (15) & Fosfonato & $\begin{array}{l}\text { Streptomyces } \\
\text { fradiae }\end{array}$ \\
\hline 2000 & Linezolideo (16) & $\begin{array}{l}\text { Oxazolidi- } \\
\text { nona }\end{array}$ & síntese \\
\hline 2003 & Daptomicina (17) & lipopeptídeo & $\begin{array}{l}\text { Streptomyces } \\
\text { roseosporum }\end{array}$ \\
\hline
\end{tabular}

marcadores específicos do gênero, como a epi-neoxalina (23), até então relatada apenas neste gênero. ${ }^{14}$

Espécies do gênero Aspergillus têm sido alvos de inúmeros estudos. A. fumigatus, cultivado em meio de cultura complexo suplementado por minerais, em fermentador de larga escala $(30 \mathrm{~L})$ por 10 dias, levou ao isolamento de sete dictopiprazinas (24 a 30). Estas substâncias apresentaram atividade inibitória do crescimento de $S$. aureus e M. luteus. ${ }^{15}$

Fungos endófitos são microrganismos que colonizam o interior de órgãos vegetais, sem causar efeito patogênico no hospedeiro. ${ }^{16} \mathrm{Em}$ contrapartida aos nutrientes recebidos da planta hospedeira, os microrganismos endófitos produzem metabólitos secundários que podem ser úteis à planta como, por exemplo, hormônios de crescimento ou antibióticos. Um estudo realizado com folhas saudáveis de Cryptocarya mandioccana, planta típica da Floresta Atlântica brasileira, levou ao isolamento de 15 fungos endófitos; dentre eles, uma espécie, Colletotrichum gloeosporioides, foi cultivada em caldo de batata dextrosado levando ao isolamento de (-)-cis-4-hidroxi-6-deoxiscitalona (31) e (4R)-4,8-di-hidroxi- $\alpha$-tetralona (32). Estas substâncias apresentaram atividade antifúngica significativa pelo teste de bioautografia contra Cladosporium cladosporioides e Cladosporium sphaerospermum, comparável com a atividade da nistatina. ${ }^{17}$ Os autores sugerem que a atividade apresentada pelos metabólitos secundários produzidos C. gloeosporioides esteja relacionada à função de proteção da planta hospedeira contra fitopatógenos, demonstrando o significado ecológico do estudo deste tipo de microrganismos.

Embora apresentando modesta atividade antibacteriana e antifúngica sobre fitopatógenos, substâncias com interessante variedade 
<smiles>CC1(C)SC2C(NC(=O)Cc3ccccc3)C(=O)N2C1C(=O)O</smiles>

2
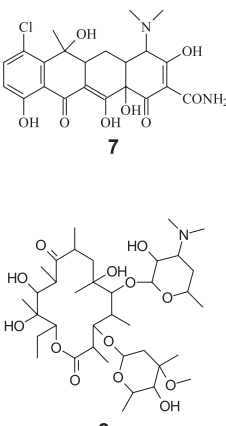

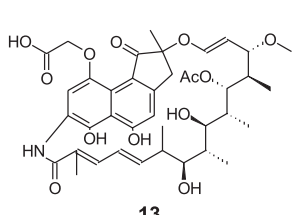

13

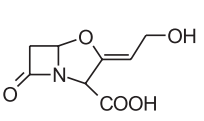

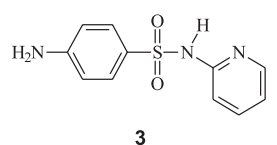

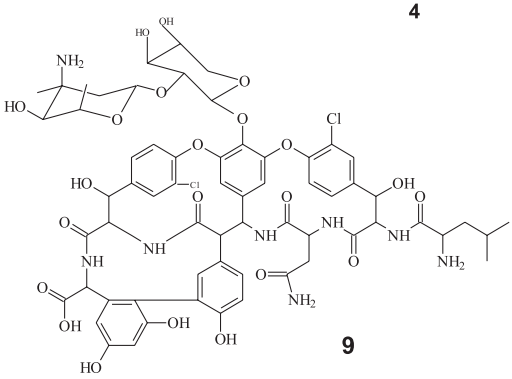

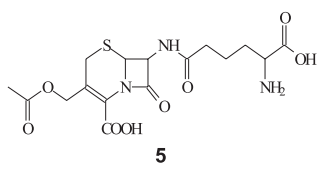
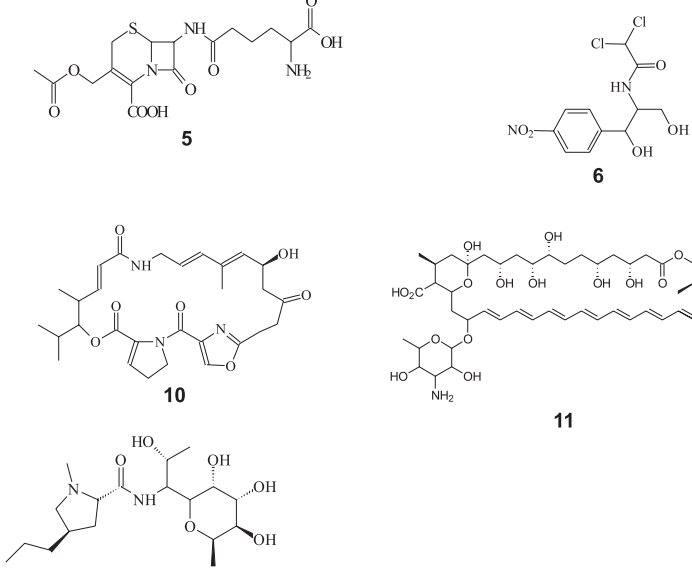

12

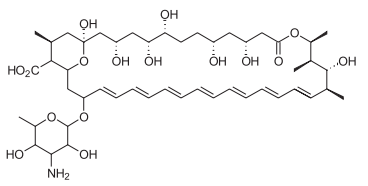

11<smiles></smiles><smiles>CC1OC1[18OH]</smiles>

15

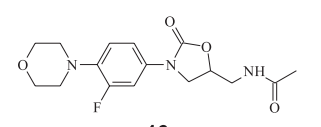

16

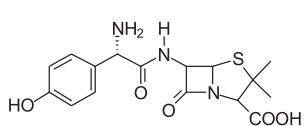

19

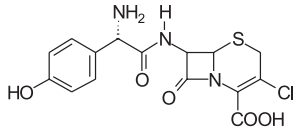

20<smiles>CO/N=C(/C(=O)NC1C(=O)N2C(C(=O)O)=C(CSc3nc(=O)c(=O)[nH]n3C)CSC12)c1csc(N)n1</smiles>

21

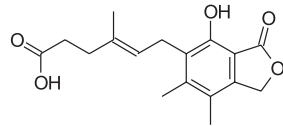

22

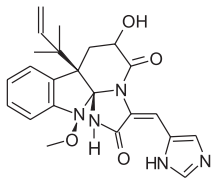

estrutural (33 a 39) foram isoladas de Microsphaeropsis olivacea, um endófito isolado de Pilgerodendron uviferum. ${ }^{18} \mathrm{O}$ estudo de Phomopsis cassiae, espécie endofítica isolada de Cassia spectabilis, levou ao isolamento de dois novos metabólitos com atividade antifúngica, ${ }^{19}$ um benzoato (40) e uma phomopsilactona (41).

Uma série de trabalhos interessantes, produzidos pelo grupo do Prof. J. B. Gloer da Universidade de Iowa (EUA), relata o estudo de esclerócios fúngicos. ${ }^{20}$ Esclerócios são estruturas produzidas por algumas espécies fúngicas visando sua sobrevivência por períodos prolongados em condições ambientais desfavoráveis. Os estudos nesta área sugerem que a presença de substâncias inseticidas em altas concentrações, especificamente em esclerócios, deve estar associada a um mecanismo de defesa, evitando que os esclerócios sejam consumidos por insetos. Na busca por substâncias inseticidas, este grupo relata o isolamento de diversas substâncias ativas também contra fungos e bactérias. O estudo de extratos provenientes de esclerócios de Penicillium raistrickii levou ao isolamento de griseofulvina (42), 6-desmetilgriseofulvina (43), além de quatro substâncias inéditas, 3,3'-di-hidroxi-6'-desmetilterfenilina (44), 3'-demetoxi-6'-desmetil5'-metoxicandidusina B (45), 6'-desmetil-candidusina B (46) e a metil-3,4,6,8-tetra-hidroxixantona (47). A substância 44 apresentou moderada atividade contra $S$. aureus e o metabólito 47 foi ativo contra B. subtilis. ${ }^{21} \mathrm{O}$ mesmo grupo, estudando $P$. griseofulvum relatou o isolamento dos sesquiterpenos penifulvinas B-E (48 a 51) e de um análogo do silfineno ${ }^{22}$ (52). Do fungo aquático Decaisnella thyridioides, Jiao e colaboradores isolaram os spironaphtalenos denominados decaspironas A-E (53 a 57), substâncias com atividades antifúngica e antibacteriana significativas. ${ }^{23}$ Interessantemente, um outro grupo, estudando uma espécie fúngica diferente (Helicoma viridis), isolou substâncias análogas (decaspironas F-I) (58 a 61), que apresentaram uma atividade modesta sobre uma cepa resistente de Pseudomonas aeruginosa. ${ }^{24}$

Espécies cropófilas exercem um intenso antagonismo entre si, competindo pelo substrato de crescimento (fezes). Nesta competição, produzem substâncias antifúngicas. Hein e colaboradores, ${ }^{25}$ estudando a espécie cropófila Ascodesmis sphaerospora, isolada a partir de fezes de bisão, relataram o isolamento de uma substância denominada arugosina F (62), devido à sua semelhança estrutural com substâncias de mesmo nome isoladas de algumas espécies de Aspergillus. Arugosina F apresentou atividade antibacteriana contra B. subtilis, $S$. aureus e antifúngica, em ensaios de competição, contra outros fungos cropófilos, Ascobolus furfuraceus e Sordaria fimicola.

Dentre os fungos saprófitas, o gênero Chaetomium é bastante representativo. Extratos da espécie $C$. cupreum, isolada na Tailândia, levaram ao isolamento dos rotiorinóis A-C (63 a 65), além da (-)-rotiorina (66), epi-isocromofilona II (67) e rubrotiorina (68), substâncias da classe das azafilonas. ${ }^{26}$ Diversos destes metabólitos são de cor vermelha, fazendo jus ao nome da espécie e da coloração que adquire o meio de cultivo deste fungo. Em ensaios de atividade antifúngica usando uma metodologia descrita por Scudiero e colaboradores, ${ }^{27}$ a substância mais ativa mostrou ser a rubrotiorina, com atividade comparável à do controle positivo utilizado, a anfotericina.

O fungo entomopatogênico Paecilomyces farinosus, isolado de larvas de insetos, foi estudado por Lang e colaboradores. ${ }^{28}$ Usando 
<smiles>[R]C1C(=O)N2CCCC2C(=O)NC1=[Y6]([H])C=Cc1ccccc1</smiles><smiles>Cc1cc(O)c(O)c2oc3cc(O)cc(O)c3c(=O)c12</smiles><smiles>CC12CCCC3(C(=O)O)C(CCC13C)C2</smiles>

52

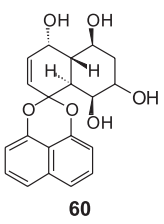

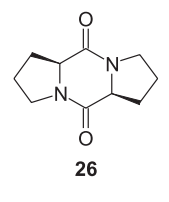<smiles>[R]C1CN2C(=O)C([R])CC2C(=O)N1</smiles>

$27 \quad R=$ i-butil; $R^{\prime}=O H$ $28 \mathrm{R}=\mathrm{i}$-propil; $\mathrm{R}^{\prime}=\mathrm{H}$ $29 \mathrm{R}=\mathrm{CH}_{2} \mathrm{Ph} ; \mathrm{R}^{\prime}=\mathrm{OH}$ $30 \mathrm{R}=$ i-butil; $\mathbf{R}^{\prime}=\mathrm{H}$

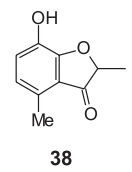<smiles>COC1=CC2(C)OC(=O)c3cc(OC)cc(O)c3C2=C(O)C1=O</smiles>

39<smiles>C=C1OC(=O)c2c(O)c(C)c(O)c(C=O)c2C1C</smiles>

41<smiles>O=C1C=COc2cccc3cccc(c23)O1</smiles>

$53 \mathrm{R}=\mathrm{H} ; \mathrm{R}^{\prime}=\mathrm{H}$ $56 \mathrm{R}=\mathrm{H} ; \mathrm{R}^{\prime}=\mathrm{OAC}$ $57 \mathrm{R}=\mathrm{OAc} ; \mathrm{R}^{\prime}=\mathrm{H}$<smiles>CCOC(=O)c1c(O)cc(O)c(C)c1C</smiles>

40

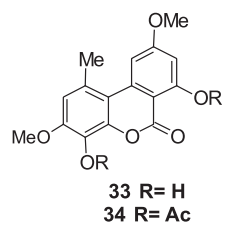

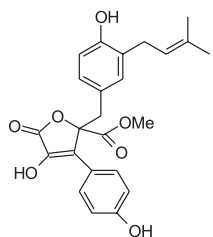

35

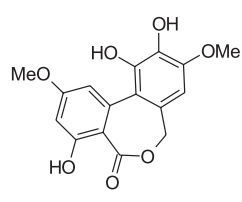

36

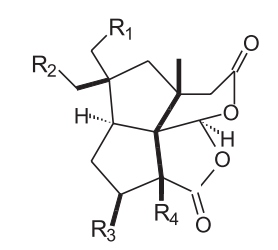

$48 \quad R_{1}=H \quad R_{2}=O H \quad R_{3}=H \quad R_{4}=H$ $49 \quad R_{1}=H \quad R_{2}=\mathrm{OH} \quad R_{3}=H \quad R_{4}=H$

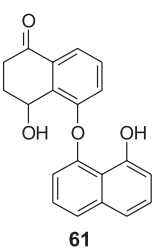
$50 \quad R_{1}=H \quad R_{2}=H \quad R_{3}=H \quad R_{4}=H$
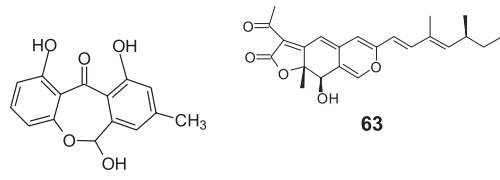<smiles>[R6]c1cc(OC)c2c(c1Cl)OC1(C(=O)OC)C(=CC(=O)CC1C)O2</smiles>
$43 \mathrm{R}=\mathrm{H}$<smiles>[R]c1c(-c2ccc(O)c(O)c2)c([R])c2c(oc3cc(O)c(O)cc32)c1[R]</smiles>

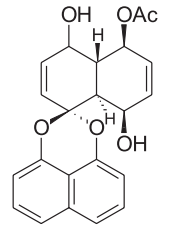

54

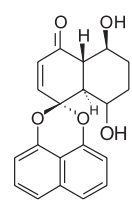

55

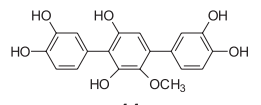

44

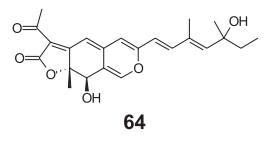

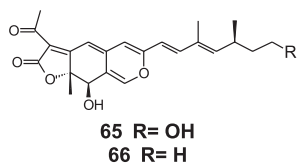

62 um protocolo muito simples de crescimento, sem agitação e em pequena escala, os pesquisadores isolaram e identificaram dois metabólitos majoritários no extrato, a N-hidroxi-2-pirona farinosona B (69) e um novo derivado do ácido tetrâmico, denominado paecilosetina (70), este último ativo contra B. subtilis, Cladosporium resinae e Trichophyton mentagrophytes. Cabrera e colaboradores, ${ }^{29}$ estudando Paecilomyces marquandii, isolaram um sorbicilinóide (71). Duas substâncias bioativas foram isoladas da espécie entomopatogênica Beauveria bassiana, um derivado furânico (72) e a (+)-bassianolona (73), substância precursora dos antibióticos cefalosporolídeos E e F. ${ }^{30}$

Fungos isolados de água ou animais e plantas marinhos têm sido menos estudados que aqueles de solo ou de origem endofítica, mas alguns relatos interessantes têm sido apresentados na literatura. Oh e colaboradores, ${ }^{31}$ estudando a espécie isolada de água fresca Massarina tunicata, isolaram três novos sesquiterpenóides, massarinolinas A-C (74 a 76). Massarinolina A mostrou atividade contra B. subtilis e $S$. aureus. O estudo químico de Dendrospora tenella, um outro fungo aquático estudado por Oh e colaboradores, ${ }^{32}$ levou ao isolamento de quatro novas substâncias denominadas ácidos tenélicos A-D (77 a 80), todas ativas contra $B$. subtilis, enquanto os ácidos 79 e 80 foram ativos também contra $S$. aureus.

Embora espécies marinhas geralmente produzam metabólitos com estruturas muito complexas, metabólitos mais simples como a naftalenona keisslona (81) isolada de Keissleriella sp YS4108 têm sido relatados. ${ }^{33} \mathrm{~A}$ keisslona foi ativa contra os fungos C. albicans, Trichophyton rubrum e A. niger.

Uma vasta bioprospecção de fungos de origem marinha foi realizada por Christophersen e colaboradores. ${ }^{34}$ As 755 espécies estudadas foram isoladas de animais, plantas e sedimentos de fontes marinhas no Mochima National Park e Paria Bay, na Venezuela e testadas contra as bactérias S. aureus ATCC 25923, E. coli ATCC 25922, Pseudomonas aeruginosa ATCC 27853 e Vibrio paraha-emolytivus ATCC 17802. 227 isolados foram caracterizados como pertencentes a gêneros ubiquitos como Eupenicillium, Penicillium, Aspergillus, Eurotium, Fusarium, Emericella, Alternaria e Gliocladium, dos quais $27 \%$ apresentaram atividade contra pelo menos uma das bactérias testadas. Em relação a uma das espécies testadas, $P$. citrinum, os autores observaram uma boa correlação entre a atividade antimicrobiana e a presença de citrinina (82), um metabólito secundário deste fungo identificado por CLAE.

Jadulco e colaboradores, ${ }^{35}$ estudando uma cepa de Curvularia lunata isolada da esponja marinha Niphates olemda coletada na Indonésia, obtiveram uma antraquinona inédita, lunatina $(\mathbf{8 3})$, além da bisantraquinona citoskirina (84). Ambas foram ativas contra $B$. subtilis, $S$. aureus e E. coli. O isolamento da antraquinona monomérica 83, junto com uma antraquinona dimérica 84 é comum e uma boa evidência de que bisantraquinonas como a citoskirina é formada pela dimerização de uma antraquinona monomérica, seguida por uma hidrogenação parcial. Neste mesmo estudo, Jadulco e colaboradores relataram o estudo de duas cepas de Cladosporium herbarum; a primeira, isolada de uma esponja marinha do gênero A. aerophoba, coletada no Mar Mediterrâneo levou ao isolamento de $\alpha$-pironas e, a segunda, isolada de uma esponja de C. aerizusa, coletada na Indonésia, levou ao isolamento de macrolídeos. Neste estudo, nenhum metabólito em comum foi isolado, embora o fungo estudado fosse o mesmo, o que é um bom exemplo de que a expressão química (metabólitos secundários) também depende do genótipo. Portanto, o reestudo de espécies de microrganismos pode levar ao isolamento de novas substâncias biologicamente ativas.

A prospecção química de Oidiodendron truncata, uma espécie fúngica isolada de uma locação extrema, o topo da montanha Endlang (4000 m) na China, foi relatada em 1999 por John e colaboradores. ${ }^{36}$ 

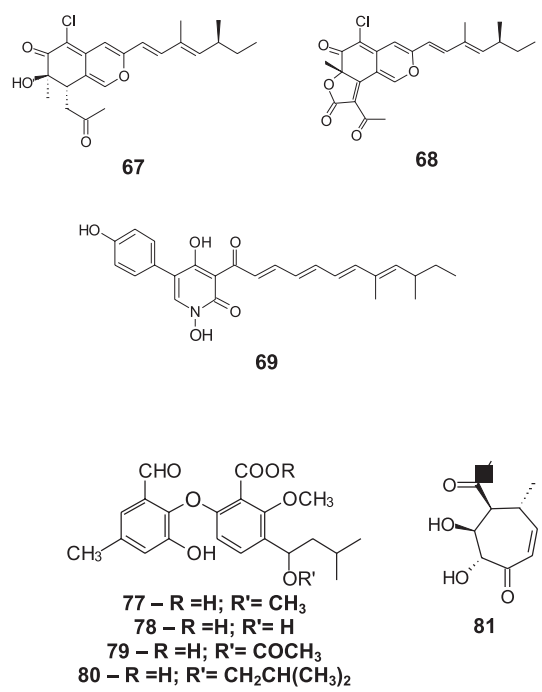

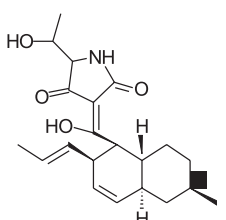

70

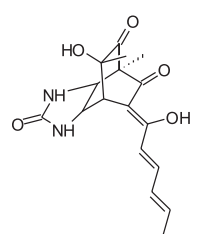

71<smiles>CC(O)CCC(=O)CC1OC(=O)CC1O</smiles>

73<smiles>[R5]C1C2CCC(C)(OC2)C1CC(O)/C=C(\C)C(=O)O</smiles>

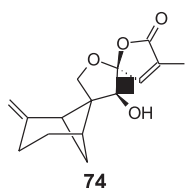<smiles>C=C1CCC2CC1C(CO)(CC(O)C=C(C)C(=O)O)C2</smiles><smiles>CC1OC=C2C(=CC(=O)C(C(=O)O)=C2O)C1C</smiles>

82

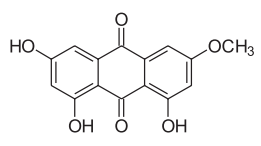

83

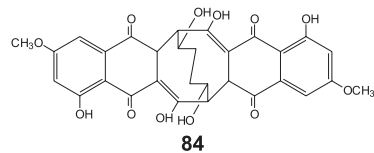<smiles>CC(O)CCC(=O)CC1OC(=O)C[C@H]1O</smiles>

72
Nesse estudo, além de dois diterpenos conhecidos, quatro novas substâncias, oidiolactonas C-F foram isoladas e suas estruturas foram determinadas por métodos espectroscópicos e análise por raios-X. Oidilactonas A (85) e B (86) mostraram moderada atividade antibacteriana contra Bacillus megaterium, além de potente atividade herbicida.

Substâncias ativas contra bactérias multirresistentes também têm sido isoladas. O oligossacarídeo Sch 58761 (87), análogo clorado da Ziracin (em desenvolvimento pela Shering-Poulgh) ${ }^{37}$ demonstrou atividade in vitro e in vivo contra cepas de Staphylococcus aureus resistentes à meticilina e contra espécies de Enterococcus resistentes à vancomicina. Sch 58761, cuja estrutura foi elucidada por criterioso estudo de fragmentação da molécula por FAB-MS e estudos espectroscópicos de NOESY e HMBC, também apresentou atividade contra diversas cepas tradicionais de organismos Gram-positivos. ${ }^{38}$ A partir de Bionectra byssicola foram isoladas as bionectinas A-C (88 a 90), ativas contra cepas de $S$. aureus resistentes à meticilina. ${ }^{39}$

A indústria farmacêutica tem se voltado ao potencial dos metabólitos fúngicos para o desenvolvimento de novos fármacos. Singh e colaboradores, da Merck Research Laboratories, relataram recentemente o isolamento de duas substâncias interessantes, as citrafunginas A (91) e B (92), com atividade sobre fungos patogênicos em baixas doses. ${ }^{40}$

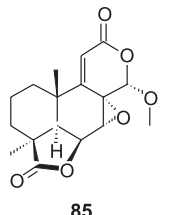

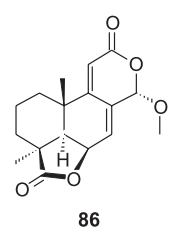

86

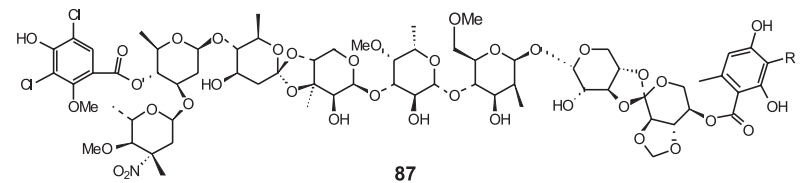

87

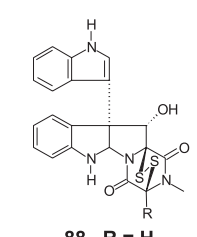

$88 \mathrm{R}=\mathrm{H}$ $89 \mathrm{R}=\mathrm{CH}(\mathrm{OH}) \mathrm{CH}_{3}$

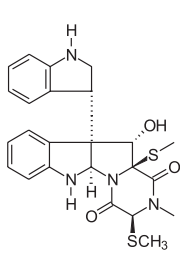

90

\section{PROCESSO FERMENTATIVO}

A produção quali e quantitativa de metabólitos secundários depende da capacidade biossintética do microrganismo e das condições de fermentação. Assim, a manipulação dos parâmetros do processo fermentativo pode alterar a expressão dos metabólitos secundários produzidos. Em geral, a biossíntese de metabólitos secundários é ativada na fase final do crescimento logarítmico ou já na fase estacionária da fermentação, quando a divisão celular e a produção de biomassa ocorrem em níveis muito baixos. ${ }^{41}$ De acordo com Tanaka, ${ }^{42}$ a produção de antibióticos por fungos tem algumas características, comuns à produção de outros metabólitos secundários: a produção de antibióticos é específica da linhagem; há uma instabilidade no processo biossintético, com tendência a uma diminuição com repiques sucessivos usados para a manutenção das linhagens; a produção de antibióticos segue a cinética de crescimento fúngico associado a um meio de cultura específico; o aumento da produção de um metabólito secundário freqüentemente ocorre na etapa de esporulação do microrganismo; a variedade estrutural dos metabólitos biossintetizados pode ser aumentada com pequenas variações do meio de cultivo.

O crescimento de microrganismos pode ocorrer em fase sólida (usualmente ágar suplementado com nutrientes específicos) ou líquida, esta última podendo ou não ser submetida à aeração. $\mathrm{O}$ uso de fermentadores específicos é desejável para se obter a reprodutibilidade do processo. Novas técnicas como a fermentação em estado (ou suporte) sólido têm surgido, no intuito de promoverem um crescimento fúngico mais rápido e uma produção diferenciada de metabólitos secundários. ${ }^{43} \mathrm{Na}$ fermentação em estado sólido, emprega-se um substrato natural como fonte de carbono, em presença de uma quantidade mínima de água. Substratos sintéticos como polímeros, com suplementação nutricional, sozinhos ou misturados a substratos naturais, também têm sido usados, desde que o material sintético seja inerte frente ao fungo alvo do processo fermentativo. A fermentação em estado sólido para a produção de antibióticos tem sido considerada muito vantajosa, com um aumento do rendimento e período fermentativo mais curto em relação a procedimentos paralelos realizados em culturas submersas. ${ }^{44}$

Os ascocarpos de Eupenicillium molle Malloch et Chain (Trichocomaceae) foram alvo de estudo por fermentação em substrato sólido (grãos de milho). Ascocarpos são corpos de frutificação especializados, análogos aos esclerótios, adaptados a condições extremas de temperatura e restrição de nutrientes e água. ${ }^{45}$ Deste trabalho, foram 

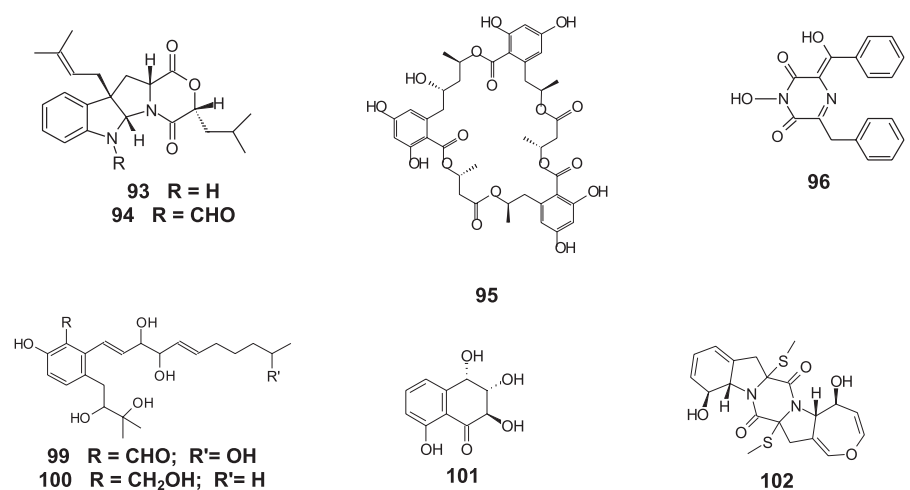

102
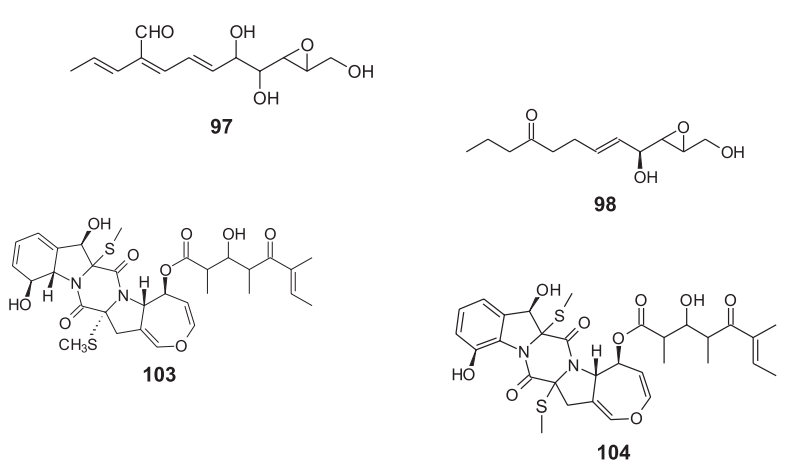

isoladas duas novas dioxomorfolinas, denominadas molleninas A (93) e B (94), respectivamente.

Um elegante estudo foi realizado por Wang e colaboradore ${ }^{45}$ para determinação da configuração absoluta da mollenina A. A configuração $16 R$ foi detectada, embora se presumisse, para este centro, a configuração $S$, proveniente de um intermediário do tipo triptofano. Novos estudos espectroscópicos sugeriram que a mollenina A se derivaria da condensação de um resíduo modificado do L-triptofano, com uma unidade do ácido $R$-2-hidroxiiso-capróico. A estereoquímica não usual de C-16 surgiria a epimerização deste centro. Embora a motivação para o estudo do extrato de E. molle tenha sido a atividade larvicida contra Helicoverpa zea, uma larva que destrói espigas de milho, as molleninas A e B não foram ativas contra esta larva, mas apresentaram atividade antibacteriana contra $B$. subtilis.

A influência das condições de cultivo na produção quali e quantitativa de metabólitos secundários pode ser bem visualizada no estudo de extratos do fungo Menisporopsis theobromae. Chinworrungsee e colaboradores $^{46}$ isolaram a menisporopsina A (95), uma polilactona macrocíclica, do cultivo de $M$. theobromae BCC4162, incubando o fungo por 7 dias a $25^{\circ} \mathrm{C}$ em BDA, posteriormente transferindo este inóculo para o meio líquido PYGM (peptona/levedura/glicose). O mesmo grupo isolou, desta espécie, em estudo posterior, ${ }^{47}$ outras nove substâncias (96 a 104) de grande diversidade estrutural usando caldo de batata dextrosado para o crescimento fúngico. Madla e colaboradores ${ }^{48}$ relataram otimizações interessantes deste processo fermentativo.

Observando as condições utilizadas para a produção de antibióticos por fungos, parece consensual que meios de cultura de constituição complexa são mais adequados que meios de cultura de constituição quimicamente definida (Tabela 2). Meios relativamente simples, como o CBD (caldo de batata dextrosado), têm apresentado resultados encorajadores.

Outras abordagens além de alterações do meio de cultura têm sido descritas objetivando-se otimizações do processo fermentativo. Por exemplo, a ação da irradiação gama sobre algumas espécies de Gliocladium spp. levou ao surgimento de cepas mutantes das quais algumas produziam níveis mais elevados de antibióticos e inibiam, mais eficazmente que a cepa de origem, o crescimento de outros fungos. ${ }^{49}$

\section{CONCLUSÃO}

O estudo que associa aspectos químicos e propriedades biológicas dos metabólitos fúngicos é, desde a década de 30, alvo de crescente interesse da comunidade científica mundial, tendo conduzido a resultados que justificaram o registro de centenas de patentes e a obtenção de medicamentos e aditivos de alimentos de grande sucesso comercial. Aliando a potencialidade de tal área de pesquisas ao fato de o nosso país possuir uma extraordinária megabiodiversidade, o interesse na exploração deste campo é amplamente justificável merecendo a atenção da comunidade científica nacional.
Tabela 2. Condições de cultivo utilizadas para a produção de metabólitos com atividade antibiótica

\begin{tabular}{|c|c|c|c|c|c|c|}
\hline $\begin{array}{l}\text { Espécie } \\
\text { fúngica }\end{array}$ & $\begin{array}{c}\text { Metabólitos } \\
\text { isolados }\end{array}$ & $\begin{array}{l}\text { Meio de } \\
\text { cultura }\end{array}$ & & $\begin{array}{l}\text { o Tempo } \\
\text { de culti- } \\
\text { vo (dias) }\end{array}$ & $\begin{array}{c}\mathrm{T} \\
\left({ }^{\circ} \mathrm{C}\right)\end{array}$ & Ref. \\
\hline $\begin{array}{l}\text { Massarina } \\
\text { tunicata }\end{array}$ & $\begin{array}{l}\text { sesquiter- } \\
\text { penos }\end{array}$ & PDB & orbital & 30 & $25-28$ & 30 \\
\hline $\begin{array}{l}\text { Dendrospora } \\
\text { tenella }\end{array}$ & $\begin{array}{c}\text { Fenóis } \\
\text { substituídos }\end{array}$ & PDB & orbital & 35 & $25-28$ & 31 \\
\hline $\begin{array}{l}\text { Paecilomyces } \\
\text { farinosus }\end{array}$ & $\begin{array}{l}\text { Ácidos } \\
\text { tetrâmicos }\end{array}$ & HSSDYB & não & 21 & 26 & 28 \\
\hline $\begin{array}{l}\text { Oidiodendron I } \\
\text { truncata }\end{array}$ & Diterpenos & $\mathrm{BM}$ & ni & 27 & 17 & 35 \\
\hline $\begin{array}{l}\text { Eupenicillium } \\
\text { mole }\end{array}$ & morfolinas & GM & não & & & 27 \\
\hline $\begin{array}{l}\text { Ascodesmis } \\
\text { sphaerospora }\end{array}$ & xantonas & PDB & orbital & 37 & $25-28$ & 25 \\
\hline $\begin{array}{l}\text { Penicillium } \\
\text { raistrickii }\end{array}$ & $\begin{array}{l}\text { Xantonas } \\
\text { e terfenilas }\end{array}$ & GM & não & & & 21 \\
\hline $\begin{array}{l}\text { Colletotrichum } \\
\text { gloeosporioides }\end{array}$ & $\begin{array}{ll}n & \text { Tetra- } \\
e s & \text { lonas }\end{array}$ & PDB & orbital & 28 & 25 & 17 \\
\hline $\begin{array}{l}\text { Decaisnella } \\
\text { thyridioides }\end{array}$ & $\begin{array}{l}\text { descapi- } \\
\text { ronas }\end{array}$ & Arroz & não & 35 & 25 & 23 \\
\hline $\begin{array}{l}\text { Paecilomyces } \\
\text { marquandii }\end{array}$ & vertinóides & YPD f & $\begin{array}{l}\text { fermen- } \\
\text { tador }\end{array}$ & 30 & 25 & 29 \\
\hline $\begin{array}{l}\text { Helicoma } \\
\text { viridis }\end{array}$ & $\begin{array}{l}\text { decaspi- } \\
\text { ronas }\end{array}$ & $\mathrm{CP}$ & orbital & 10 & 25 & 24 \\
\hline $\begin{array}{l}\text { Menisporopsis } \\
\text { theobromae }\end{array}$ & $\begin{array}{l}\text { Menispo- } \\
\text { ropsinas }\end{array}$ & FEC & orbital & $4-28$ & 25 & 48 \\
\hline
\end{tabular}

theobromae ropsinas

$\overline{\mathrm{BM}}=$ Biomalte, $5 \% ; \mathrm{CO}=$ complexo, suplementado com óleo; $\mathrm{CP}$ $=$ complexo, contendo glicose, amido, soja, levedura e milho; $\mathrm{FEC}=$ $1 \%$ frutose, 2,5\% extrato de carne; GM: grãos de milho; HSSDYB = Caldo Sabouraud-dextrose-levedura, "meia-força" (diluído 1:1 em água em relação à concentração padrão dos reagentes); $\mathrm{CBD}=$ Caldo de batata dextrosado; $\mathrm{YPD}=0,5 \%$ extrato de levedura bacteriológica, $1 \%$ de peptona bacteriológica, $1 \%$ dextrose em água do mar artificial; ni= não informado

Outras informações sobre o assunto podem ser encontradas na literatura. Uma excelente revisão sobre metabólitos secundários de fungos marinhos, cobrindo 273 moléculas isoladas destes organismos e suas respectivas atividades biológicas foi publicada por Bugni e colaboradores ${ }^{50}$ Gunatilaka ${ }^{16}$ reuniu dados como distribuição, diversidade estrutural e bioatividade de microrganismos endofíticos. $\mathrm{O}$ mesmo tema foi mais brevemente descrito por Strobel e Daisy. ${ }^{51}$

Revisões mais específicas também estão disponíveis. Quang e colaboradores ${ }^{52}$ descrevem o isolamento, identificação e bioprospecção (atividades antioxidante, antifúngica, alelopática e citotóxica) de 
fungos da família Xylariaceae, incluindo uma discussão quimiossistemática dos gêneros Daldinia e Hypoxylon.

Enquanto soluções mais efetivas para o problema da resistência bacteriana, que poderão advir da descoberta de novas drogas através de ferramentas da genômica, não surgem, a bioprospecção de produtos naturais, inclusive fúngicos, continua sendo uma poderosa aliada no processo de descoberta de novos antibióticos.

\section{AGRADECIMENTOS}

Aos membros do Laboratório de Biotecnologia e Bioensaios do Departamento de Química da UFMG, à FAPEMIG e International Foundation for Science.

\section{REFERÊNCIAS}

1. Gainey, P. L.; J. Agric. Res. 1917, 10, 355.

2. Korolkovas, A.; Burkhalter, J. H.; Química Farmacêutica, GuanabaraKoogan: Rio de Janeiro, 1988.

3. Strohl, W. R.; Biotechnology of antibiotics, $2^{\text {nd }}$ ed., Marcel Dekker, Inc: New York, 1997.

4. Barrett, C. T.; Barrett, J. F.; Curr. Opin. Biotechnol. 2003, 14, 621.

5. Butler, M. S.; J. Nat. Prod. 2004, 67, 2141.

6. Zhang, W.; Becker, D.; Cheng, Q.; Recent Pat. Anti-Infect. Drug Discovery 2006, 1, 224.

7. Oliveira, I. S.; Moura, R.. M.; Luz, E. D. M. N.; Fitopatol. Bras. 2006, 31,408 .

8. Santos, G. R; Café-Filho, A. C.; Reis, A.; Fitopatol. Bras. 2006, 31, 476.

9. Aremu, E. A.; Tanaka, K.; Akagi, Y.; Maekawa, N ; Akamatsu, H.; Kodama, M. H.; J. Gen. Plant Patol. 2004, 70, 139

10. Walshi, C. T.; Wright, G.; Chem. Rev. 2005, 105, 391.

11. Berdy, J.; The discovery of new bioactive microbial metabolites: screening and identification. Bioactive metabolites from microorganisms, $27^{\text {th }}$ ed., Elsevier: London, 1989.

12. Newman, D. J.; Cragg, G. M.; J. Nat. Prod. 2007, 70, 461.

13. Vinokurova, N. G.; Ivanushkina, N. E.; Kochikina, G. A.; Aribasarov, M. U.; Ozerskaya, S. M.; Appl. Biochem. Microbiol. 2005, 41, 83.

14. Overy, D. P.; Phippis, R. K.; Frydenvang, K.; Larsem, T. O.; Biochem. Syst. Ecol. 2006, 34, 345.

15. Furtado, N. A. J. C.; Pupoa, M. T.; Carvalho, I.; Campoa, V.; Duarteb, M. C. T.; Bastos, J. K.; J. Braz. Chem. Soc. 2005, 16, 1448.

16. Gunatilaka, A. A. L.; J. Nat. Prod. 2006, 69, 509.

17. Inácio, M. L.; Silva, G. H.; Teles, H. L.; Trevisan, H. C.; Cavalheiro, A. J.; Bolzani, V. S.; Young, J.; J. Agric. Res. 2006, 10, 355.

18. Hormazabal, E.; Schmeda-Hirschmann, G.; Astudillo, L.; Rodriguez, J.; Theoduloz, C.; J. Biosci. Bioeng.. 2005, 60, 11.

19. Silva, G. H.; Teles, H. L.; Trevisan, H. C.; Bolzani, V. S.; Young, M. C. M.; Pfenning, L. H.; Eberlin, M. N.; Haddad, R.; Costa-Neto, C. M.; Araújo, A. R.; Chem. Soc. 2005, 16, 1463.

20. Oh, H. B.; Gloer, J.; Wicklow, D. T.; Dowd, P. F.; J. Nat. Prod. 1998, 61, 702 .

21. Belofsky, G. N.; Gloer, J. B.; Wicklow, D. T.; Dowd, P. F.; J. Nat. Prod. 1998, 61, 1115.

22. Shim, S. H.; Gloer, J. B.; Wicklow, D. T.; J. Nat. Prod. 2006, 69, 1601.

23. Jiao, P.; Swenson, D. C.; Gloer, J. B.; Campbell, J.; Shearer C. A.; J. Nat. Prod. 2006, 69, 1667.

24. Hu, H.; Guo, H.; Li, E.; Liu, X.; Zhou, Y.; Che, Y.; J. Nat. Prod. 2006,
69, 1672.

25. Hein, S. M.; Gloer, J. B.; Koster, B.; Malloch, D.; J. Nat. Prod. 1998, 61,1566 .

26. Kanokmedhakul, S.; Kanokmedhakul, K.; Nasomjai, P.; Louangsysouphanh, S.; Soytong, K.; Isobe, M.; Kongsaeree, P.; Prabpai, S.; Suksamrarnr, A.; J. Nat. Prod. 2006, 69, 891.

27. Scudiero, D. A.; Shoemaker, R. H.; Paull, K. D.; Monka, A.; Tierne, S.; Nofziger, T. H.; Currens, M. J.; Seniff, D.; Boyd, M. R.; Cancer Res. 1988, 48, 4827.

28. Lang, G.; Blunt, J. W.; Cummings, N. J.; Cole, A. L. J.; Munro, M. H. G.; J. Nat. Prod. 2005, 68, 810 .

29. Cabrera, G. M.; Butler, M.; Rodriguez, A. M.; Godeas, A.; Haddad, R.; Eberlin, M. N.; J. Nat. Prod. 2006, 69, 1806.

30. Oller-Lopez, J. 1.; Iranzo, M.; Mormeneo, S.; Oliver, E.; Cuerva, J. M.; Oltra, J. E.; Org. Biomol. Chem. 2005, 3, 1172.

31. Oh, H.; Gloer, J. B.; Shearer, C. A.; J. Nat. Prod. 1999, 62, 497.

32. Oh, H.; Kwon, T. O.; Gloer, J. B.; Marvanova, L.; Shearer, C. A.; J. Nat. Prod. 1999, 62, 580.

33. Liu, C. H.; Liu, J. Y.; Huang, L. L.; Zou, W. X.; Tan, R. X.; Planta Med. 2003, 69, 481 .

34. Christophersen, C.; Crescente, O.; Frisvad, J. C.; Gram, L.; Nielsen, J.; Nielsen, P. H.; Rahbaek, L.; Mycopatology 1999,143, 135.

35. Jadulco, R.; Brauers, G.; Edrada, R. A.; Ebel, R.; Wray, V.; Sudarsono, P.; Proksch, P.; J. Nat. Prod. 2002, 65, 730.

36. John, M.; Krohn, K.; Flörke, U.; Aust, H-J.; Draeger, S.; Schulz, B.; J. Nat. Prod. 1999, 62, 1218.

37. Poulet, F.; Veneziale, R.; Vancutsem, P.; Losco, P.; Treinen, K.; Morrissey, R.; Toxicol. Pathol. 2005, 33, 320.

38. Chu, M.; Mierzwa, R.; Patel, M.; Jenkins, J.; Das, P.; Pramanik, B.; Chan, T-M.; Tetrahedron Lett. 2000, 41, 6689.

39. Zheng, C.; Kim, C.; Bae, K. S.; Kim, Y.; Kim, W.; J. Nat. Prod. 2006, 69, 1816.

40. Singh, S. B.; Zink, D. L.; Doss, G. A.; Polishook, J. D.; Ruby, C.; Register, E.; Kelly, T. M.; Bonfiglio, C.; Willianson, J. M.; Kelly, R.; Org. Lett. 2004, 6, 337.

41. Yarbrough, G. G.; Taylor, D. P.; Rowlands, R. T.; Crawford, M. S.; Lasure, L. L.; J. Antibiot. 1993, 46, 535.

42. Tanaka, M.; Hori Y.; Ueda, H.; Ezaki, M.; Goto, T.; Masashi, H.; Okuhara, M.; J. Antibiot. 1993, 46, 858.

43. Asagbra, A. E.; Sanni, A. I.; Oyewole, O. B.; World J. Microbiol. Biotechnol. 2005, 21, 107.

44. Mizumoto, S.; Hirai, M.; Shoda, M.; Appl. Microbiol. Biotechnol. 2006, 72,869

45. Wang, H-J.; Gloer, J. B.; Wicklow, D. T.; Dowd, P. F.; J. Nat. Prod. 1998, 61, 804

46. Chinworrungsee, M.; Kittakoop, P.; Isaka, M.; Maithip, P.; Supothia, S.; Thebtaranonth, Y.; J. Nat. Prod. 2004, 67, 689.

47. Chinworrungsee, M.; Kittakoop, P.; Saenboonrueng, J.; Kongsaeree, P.; Thebtaranonth, Y.; J. Nat. Prod. 2006, 69, 1404.

48. Madla, S.; Kittakoop, P.; Wongsa, P.; Lett. Appl. Microbiol. 2006, 43, 548.

49. Mohamed, H. A.; Haggag, W. M.; Bull. National Res. Centre (Egypt) 2005, 30, 231.

50. Bugni, T. S.; Ireland, C. M.; Nat. Prod. Rep. 2004, 21, 143.

51. Strobel, G.; Daisy, B.; Microb. Molec. Biol. Rev. 2003, 64, 491.

52. Quang, D. N.; Hashimoto, T.; Asakawa, Y.; Recent Res. Develop. Phytochem. 2005, 9, 1. 